\title{
Ultrasound-guided spermatic cord block for scrotal surgery
}

\author{
M. Wipfli ${ }^{1}$, F. Birkhäuser ${ }^{2}$, C. Luyet $^{1 *}$, R. Greif ${ }^{1}$, G. Thalmann ${ }^{2}$ and U. Eichenberger ${ }^{1}$ \\ ${ }^{1}$ University Department of Anaesthesiology and Pain Therapy and ${ }^{2}$ Department of Urology, University Hospital and University of Berne, \\ Berne, Switzerland \\ *Corresponding author. E-mail: cedric.luyet@insel.ch
}

\section{Editor's key points}

- Spermatic cord block for scrotal surgery can be an alternative to neuraxial and general anaesthesia and provides long-lasting postoperative analgesia; however, major limitations of the conventional anatomical landmark-based blind injection technique limit its use.

- This study showed that a new ultrasound-guided technique for spermatic cord block is feasible and has a high success rate.
Background. Performing spermatic cord block for scrotal surgery avoids the potential risks of neuraxial and general anaesthesia and provides long-lasting postoperative analgesia. A blindly performed block is often inefficient and bears its own potential risks (intravascular injection of local anaesthetics, haematoma formation and perforation of the deferent duct). The use of ultrasound may help to overcome these disadvantages. The aim of this study was to test the feasibility and monitor the success rate of a new ultrasoundguided spermatic cord block.

Methods. Twenty consecutive patients undergoing urologic surgery (subcapsular orchiectomy or vaso-vasostomy) were included in this prospective study. Using a linear ultrasound probe, the spermatic cord was identified by locating the spermatic artery and the deferent duct. A $23 \mathrm{G} \mathrm{Microlance} \mathrm{needle} \mathrm{was} \mathrm{advanced} \mathrm{close} \mathrm{to} \mathrm{the} \mathrm{deferent} \mathrm{duct} \mathrm{by}$ avoiding vessel perforation, and local anaesthetic was deposited around the deferent duct under direct visualization. The primary outcome was the success rate of the block which was defined as surgery without any substitution of opioids, additional local anaesthetics, or sedatives.

Results. In 20 patients, 40 blocks were performed with a success rate of $95 \%(n=38)$. The failure rate was $5 \%(n=2)$ and no conversion to general anaesthesia was needed. The mean duration of the block was $14.1 \mathrm{~h}$ (SD 6.9).

Conclusions. The use of ultrasound guidance to perform spermatic cord block is feasible and has a high success rate. Our new approach may become a suitable alternative to neuraxial or general anaesthesia especially in the ambulatory surgical setting.

Registry: International Standard Randomised Controlled Trial Number Register; www. controlled-trials.com; Registry Nr.: ISRCTN44647819.

Keywords: anaesthetic techniques, regional; anaesthetics local; equipment, ultrasound machines; regional anaesthesia; surgery, urological

Accepted for publication: 18 September 2010
The sensory innervations of the region between the abdomen and the inner surface of the thigh, which includes the groin and the testes, are supplied by the three 'border nerves'. ${ }^{1}$ The iliohypogastric and ilioinguinal nerves originate from the lumbar plexus (12th thoracic to second lumbar roots). After passing together laterally through the psoas muscle and extending diagonally along the ventral surface of the lumbar quadrate muscle, they run more caudally, piercing the three abdominal wall muscles (transverse abdominal, internal oblique, and external oblique muscles) near the iliac crest to continue towards the deep inguinal ring into the inguinal canal. The third nerve innervating the region, the genitofemoral nerve, originates from the first and second lumbar roots and passes retroperitoneally over the psoas muscle. More distally, it divides into femoral and genital branches.

The genital branch of the genitofemoral nerve and the terminal branch of the ilioinguinal nerve innervate together the sensory part of the scrotal content. ${ }^{2}$ After emergence through the external ring of the inguinal canal, both nerves are found inside the spermatic cord either on top or under the cremasteric fascia. At their emergence at the external ring, they are readily accessible for pharmacological block. ${ }^{2}$ It is important to emphasize that a block of these two nerves will not provide effective analgesia to the scrotal skin but only to the scrotal content. Since the sensory innervations of the skin are provided by the branches of the pudendal nerve, an additional infiltration of the skin, locally at the side of incision, is mandatory. ${ }^{2-5}$

Scrotal surgery with spermatic cord block without the need of general or neuraxial anaesthesia has potentially cost-saving effects, especially in the outpatient setting. ${ }^{3}$ Furthermore, it has the potential of fewer side-effects and better postoperative analgesia compared with general or neuraxial anaesthesia. ${ }^{6}{ }^{7}$ Ezeh and colleagues could demonstrate that median postoperative pain intensity, sedation 
score, and analgesic requirements were significantly lower after spermatic cord block compared with general anaesthesia for testicular sperm extraction. These advantages led to a shorter recovery time, less expensive care, and greater patient satisfaction. ${ }^{6}$ In a multicentre randomized trial, Nordin and colleagues compared local, neuraxial, and general anaesthesia in groin hernia repair surgery: patients in the local anaesthesia group had a shorter hospital stay, less postoperative pain, and fewer voiding difficulties compared with patients in the neuraxial or general anaesthesia group. ${ }^{7}$

Until now, spermatic cord block was exclusively performed with a blind, conventional anatomical landmark-based method. $^{2-6} 8-10$ Because of the presence of multiple vessels in the spermatic cord (pampiniform plexus and testicular arteries), the blind technique is associated with the possibility of an intravasal local anaesthetic injection with the danger of systemic intoxication. The perforation and damage of the testicular artery with the potential of subsequent bleeding and haematoma formation is also a well-described serious side-effect of the blind technique. ${ }^{11}$ Furthermore, the blind injection is often painful and can lead to persistent pain at the site of injection. ${ }^{2-6}$ Also the difficulty of defining the pubic tubercle-especially in obese individuals-requires additional injection of local anaesthetics into the spermatic cord after the beginning of surgery in a lot of patients. $^{8}$

These major limitations of the conventional anatomical landmark-based blind injection technique limit the use of the spermatic cord block today. One possible solution to solve the problems may be the visualization of the structures and the injection under sight. Ultrasound-guided regional anaesthesia is a widely accepted method in the rapidly evolving field of regional anaesthesia. Ultrasound guidance for peripheral nerve blocks improves block success rates, has faster onset, longer duration, decreases the risk of vascular puncture during block performance compared with electrical neurostimulation, and may decrease block-related complications. $^{12} 13$

The aim of this prospective, consecutive case series was to test the feasibility of ultrasound-guided spermatic cord block and to monitor its success rate.

\section{Methods}

After obtaining ethical approval from the ethics committee of the Canton Berne and written consent, we included 20 consecutive patients undergoing scrotal surgery either for vaso-vasostomy or subcapsular orchiectomy in the study. The study was performed at the Department of Anaesthesiology and Pain Therapy in collaboration with the Department of Urology at the University Hospital of Bern. Exclusion criteria were refusal of regional anaesthesia, adiposity with a BMI over 40, or known bleeding disorders and blood coagulation abnormalities as INR $>1.5$ or platelet count under $100000 \mathrm{\mu l}^{-1}$.
After standard patient monitoring (non-invasive arterial pressure measurement, electrocardiography, oxygen saturation), insertion of a peripheral i.v. line and administration of 2 litre $\min ^{-1}$ of nasal oxygen and an i.v. bolus of midazolam (0.01-0.03 $\mathrm{mg} \mathrm{kg}^{-1}$ ) were done. Thereafter, the groin region was shaved and disinfected with Betaseptic ${ }^{\text {TM }}$ (Mundipharm Medical Company, Hamilton, Bermuda).

\section{Block placement using ultrasound}

The spermatic cord and its contents were visualized by ultrasound (M-Turbo, SonoSite Inc., Bothell, WA, USA) using a linear array transducer (L25x, 13-6 MHz, $25 \mathrm{~mm}$ broadband linear array) at the right and left inguinoscrotal junction distal to the external ring of the inguinal canal. Sterility was provided by covering the probe with a sterile dressing (Tegaderm $^{\mathrm{TM}}$, Ref $1626 \mathrm{~W}, 10 \times 12 \mathrm{~cm}, 3 \mathrm{M}$ Heath Care, Neuss, Germany) and using sterile ultrasound gel (Aquosonic 100, Parker Laboratories Inc., Fairfield, NJ, USA). We found the four-hand method by pulling the spermatic cord gently to the surface with the thumb and the index finger of a helping person to be the best way to visualize the anatomical structures with ultrasound (Fig. 1).

At each site, the spermatic cord was identified as a halfrounded structure surrounded by the external spermatic fascia and its contents. Inside the spermatic cord, the testicular artery was first identified by Doppler ultrasound. The deferent duct was easy to discern as a round noncompressible anatomical structure with no Doppler flow signal (Fig. 2). After a further disinfection of the puncture site, a 23G Microlance ${ }^{T M}$ sharp needle (Becton Dickinson AG, Fraga, Spain) was inserted under out-of-plane real-time ultrasound guidance with the spermatic cord in short axis and directed towards the deferent duct, contralaterally to the testicular artery. The needle tip was advanced to direct contact with the deferent duct. That made it possible to visualize the local anaesthetic spreading around the

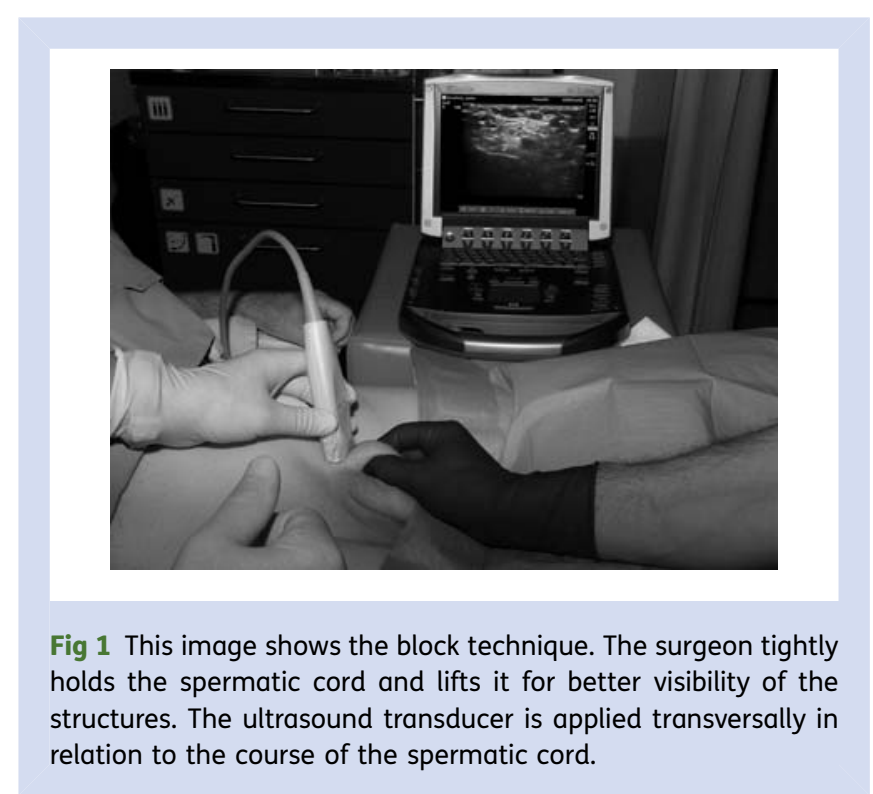




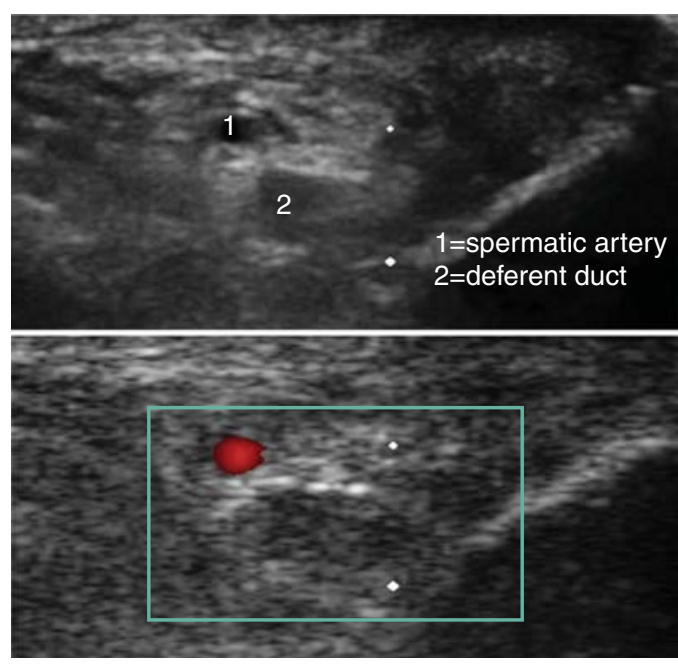

Fig 2 The spermatic cord with its contents. The deferent duct is visible as a non-compressible, internal hypoechoic structure with hyperechoic horizon. The venous plexus is not visible due to the compression of the structures with the pinging method as visible in Figure 1. The upper image shows the spermatic cord without Doppler signal. By adding the Doppler signal (lower image), the spermatic artery can be identified and discerned from the deferent duct, which has no flow. The aim is to inject the local anaesthetics into the spermatic cord, close to the deferent duct by avoiding the puncture of the artery. 1 , spermatic artery; 2 , deferent duct.

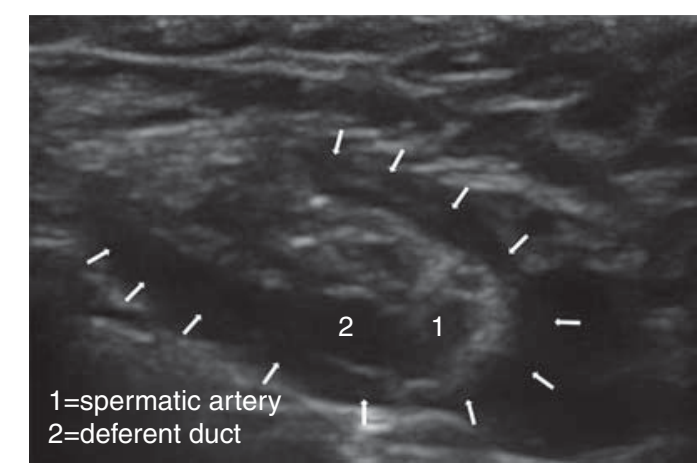

Fig 3 Spermatic cord after local anaesthetics (LA) injection. The arrows mark the hypoechoic appearing LA inside the spermatic cord, around, and between the spermatic artery and duct. 1, spermatic artery; 2, deferent duct.

deferent duct (Fig. 3). For the block, we used a mixture of 5 $\mathrm{ml}$ mepivacaine 2\% (Institute of Pharmacology, University Hospital Berne, Switzerland) and $5 \mathrm{ml}$ ropivacaine $0.75 \%$ (AstraZeneca AG, Zug, Switzerland). Each spermatic cord was anaesthetized with $10 \mathrm{ml}$ of this mixture and the scrotal skin was infiltrated with the same anaesthetic mixture (in total 2-3 $\mathrm{ml}$ ) at the site of the incision immediately before surgery started as described by Wakefield and colleagues. ${ }^{10}$

\section{Measurements and data analysis}

Patient characteristic and morphometric data were recorded (age and BMI), and diagnosis, medication, date and time of surgery, duration of surgery, and indication for surgery.

Non-invasive arterial pressure, pulse, and ventilatory frequency were obtained every $5 \mathrm{~min}$ throughout the block and during surgery, and $\mathrm{Sa}_{\mathrm{O}_{2}}$ was monitored continuously.

\section{Primary outcome measurement}

The primary outcome was the success rate of the block, which was defined as painless surgery [visual analogue pain scale $($ VAS) $<3$ ] after ultrasound-guided spermatic cord block without any substitution of opioids, additional local anaesthetics, or conversion into general or neuraxial anaesthesia (no laryngeal mask, no tracheal intubation, no spinal anaesthesia).

\section{Secondary outcomes measurements}

(i) VAS (where 0 equals no pain and 10 equals the worst imaginable pain) 5 min before block (baseline), VAS during injection of the local anaesthetics, VAS 15 min after the beginning of surgery and every 30 min until the end of surgery.

(ii) The number of inadvertent vascular punctures during ultrasound-guided spermatic block assessed by aspiration of blood or missing fluid expansion of the cord after $0.5 \mathrm{ml}$ of injected local anaesthetic (=test dose).

(iii) Duration of the block, which was defined as time (in hours after block placement) for the first patient requirement for postoperative analgesics. If no analgesic was needed the first $24 \mathrm{~h}$, the duration was set to $24 \mathrm{~h}$.

(iv) The number of patients reporting postoperative nausea and vomiting in the first $24 \mathrm{~h}$ after the surgical procedure.

(v) The total number of intraoperative spermatic cord haematomas assessed by the surgeon during surgical preparation.

(vi) Overall patient satisfaction with the spermatic cord block as the method of anaesthesia 1 week after surgery, evaluated by telephone interview, pointed out as follows: 1 , very satisfactory; 2 , satisfactory; 3 , indifferent; 4, unsatisfactory; 5 , very unsatisfactory.

(vii) The total number of haematomas over the injection site 1 week after operation (telephone interview).

\section{Results}

In the 20 consecutive patients, a total of 40 ultrasoundguided spermatic cord blocks were performed. Details of the morphometric data and the surgical procedures are shown in Table 1. 
Table 1 Patient characteristic and perioperative data. Means (range)

$$
\mathbf{n}=\mathbf{2 0}
$$

Age (yr)

$68.2(34-90)$

BMI

$25.7(21-28)$

ASA risk classification status

$2.2(1-3)$

Surgery time vaso-vasostomy (min)

$146(135-180)$

Surgery time orchiectomy (min)

Table 2 Results of ultrasound-guided spermatic cord block. Data are numbers or means (range) if not otherwise stated. $n$, number

\begin{tabular}{ll}
\hline Total number of patients & 20 \\
Number of patients with block for orchiectomy & 12 \\
Number of patients with block for vaso-vasostomy & 8 \\
Total number of blocks & 40 \\
Success rate [\% ( $n$ )] & $95(38)$ \\
Failure rate [\% ( $n$ )] & $5(2)$ \\
Mean midazolam dose for premedication (mg) & $2.3(0-3)$ \\
Number of haematoma at injection site 1 week after & None \\
operation & \\
\hline
\end{tabular}

\section{Primary outcome measurement}

The success rate was $95 \%$ (38 of 40 blocks, Table 2). One patient undergoing vaso-vasostomy complained of pain (VAS 5) during a short time of 3-5 min, $45 \mathrm{~min}$ after the beginning of surgery on the left side. A single bolus of 250 $\mu \mathrm{g}$ i.v. alfentanil alleviated the short period of pain. Shortly afterwards, the surgery was continued without further pain or other analgesic substitution. The second patient with failed block had VAS 8, 10 min after the beginning of right subcapsular orchiectomy, received an analgosedation with a total of $15 \mathrm{mg}$ of ketamine and $65 \mathrm{mg}$ of propofol administered continuously over the remaining time of surgery. These two patients were excluded from further VAS analysis. Except of these two failed blocks, none of the patients requested supplemental analgesia.

\section{Secondary outcome measurements}

The pre- and intraoperative VASs are shown and summarized in Figure 4. No signs of vascular puncture or inadvertent intravascular injection were detected during any of the procedures. The mean duration of the block was $14.1 \mathrm{~h}$ (range, 8-24). Five patients $(25 \%)$ did not need any analgesics in the $48 \mathrm{~h}$ until hospital discharge. None of the patients showed nausea and vomiting during the first 24 postoperative hours.

No complications could be directly attributed to the spermatic cord block. One patient undergoing vaso-vasostomy showed a small locally limited haematoma of the spermatic cord which was detected during surgical preparation. This haematoma did not affect the intra- and postoperative patient management. There was no evidence of intravascular instillation of the local anaesthetics and no untoward

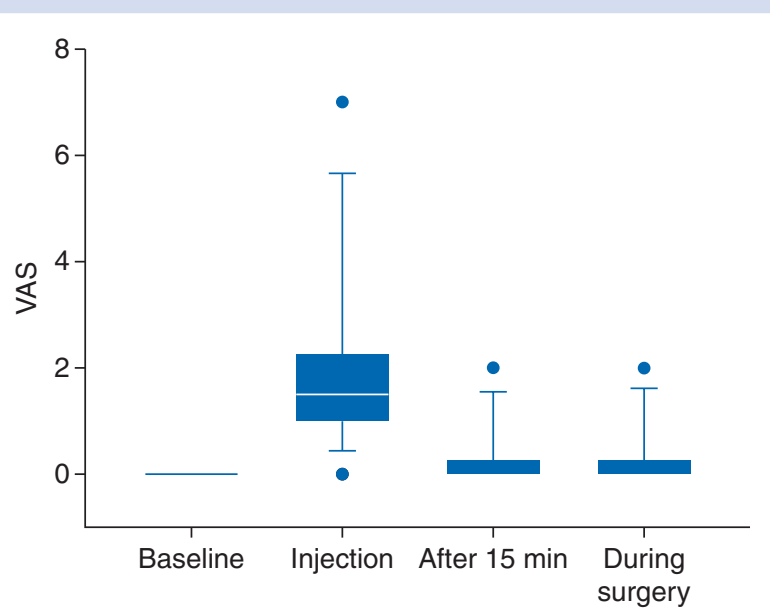

Fig 4 VAS scores at different time points. Baseline, 5 min before block; injection, during injection of the LA; after $15 \mathrm{~min}, 15 \mathrm{~min}$ after the start of surgery; during surgery, mean of VAS scores taken every 30 min until the end of surgery. The box is marked by the first quartile (25th), third quartile (75th), and median. Whiskers show the 10 th and the 90 th percentiles. Points mark the range.

reactions were recognized. No significant haemodynamic or respiratory changes were observed throughout the surgical procedures.

Two older patients, both $85 \mathrm{yr}$ old, undergoing bilateral subcapsular orchiectomy, showed agitation 15-35 min after administration of the local anaesthetic and were successfully treated with 40-60 $\mathrm{mg}$ propofol each.

All patients could be contacted 1 week after the procedure for the telephone interview. No patient complained of pain or detected haematoma or signs of infection at the site of injections. The overall mean satisfaction score was 1.2 (range, $0-2$ ). Sixteen patients scored extremely satisfactory and only four patients scored satisfactory. No one, even the two patients with failed block, would prefer another technique for anaesthesia if scrotal surgery should be required again.

\section{Discussion}

With this prospective, consecutive case series of the use of real-time ultrasound guidance to perform spermatic cord block, we showed that our new technique is feasible and effective for vaso-vasostomy and subcapsular orchiectomy.

Several blind, landmark-based spermatic cord blocks have been described in detail and are currently in use for vaso-vasostomy, hydrocelectomy, orchiectomy, and spermatocelectomy. ${ }^{2-6} 8-10$ However, all these blind approaches have different major drawbacks. First, the blind techniques are associated with the danger of intravasal injection of the local anaesthetic, leading to systemic intoxication or perforation and damage of the testicular artery with the potential of subsequent bleeding and haematoma formation. ${ }^{11}$ Secondly, the pubic tubercle-often used as an anatomical landmark in blind approaches-is difficult to identify 
especially in obese patients and may lead to a less effective block requiring additional injection of local anaesthetics into the spermatic cord during the surgical procedure. ${ }^{8}$ Thirdly, persistent pain at the site of infiltration of the local anaesthetic is a major limitation of blind approaches. ${ }^{24}$

All the mentioned drawbacks related to the blind approaches may be avoided effectively by direct visualization of the structures using ultrasound. Ultrasound guidance, introduced several years ago to perform peripheral nerve blocks, improves block success rates, leads to faster onset and longer duration of the block, and decreases the risk of vascular puncture during block as shown in a recently published review and meta-analysis of randomized controlled trials comparing ultrasound guidance with a nerve stimulator-guided (blind) puncture. ${ }^{12}$ Furthermore, ultrasound guidance is minimizing the number of needle passes required to complete the block. ${ }^{14}$ By using ultrasound, we were able to directly visualize the spermatic cord in all patients and were therefore not dependent on sometimes difficult to find anatomical landmarks as the pubic tubercle to localize the target indirectly. Thirty-eight of our 40 blocks were successful, which means the patients underwent painless surgery without any substitution by opioids or sedatives (after premedication with midazolam 0.01-0.03 mg $\mathrm{kg}^{-1}$ ). Furthermore, in the two patients with a non-successful block at one side, surgery could be terminated with only minimal supplemental analgesia and sedation and no need for conversion into general or neuraxial anaesthesia. Especially in the aged population of patients undergoing subcapsular orchiectomy, this circumstance may provide the potential of saving costs and lower the overall anaesthetic risk.

The block was well tolerated, and 16 out of the 20 patients were reported to be extremely satisfied with the anaesthesia. With one exception (a small local haematoma, detected by the surgeon after opening the spermatic cord), no complication occurred.

By using Doppler ultrasound, we could visualize the pulsatile flow of the spermatic artery in all patients, locate this vessel, and avoid its puncture under sight. Not injuring this artery is especially important in patients not undergoing subcapsular orchiectomy (in our study, the patients undergoing vasovasostomy), and in these patients, the new ultrasound-guided approach may increase the safety of the procedure-unless our study is too small to definitely conclude in this direction.

Our study has several limitations. It was a consecutive case series without a control group and without the power to compare endpoints such as complications like haematoma or other surgical outcome measurements. Because of the rather small number of 40 consecutive blocks, no definite statements regarding safety of the block can be formulated. Further studies with larger numbers of participants should confirm our results and investigate possible drawbacks of the described ultrasound method.

In conclusion, we could demonstrate the efficacy of our novel, easy-to-perform ultrasound-guided spermatic cord block. This technique might be an alternative to the often painful and ineffective blind techniques for spermatic cord block or may replace more invasive neuraxial or general anaesthesia procedures.

\section{Acknowledgement}

Special thanks to Jeff Crowder for proofreading the English of this manuscript.

\section{Conflict of interest}

None declared.

\section{Funding}

This study was supported by an institutional research grant of the University Department of Anaesthesiology and Pain Therapy, University Hospital and University of Berne, Berne, Switzerland.

\section{References}

1 Rab M, Ebmer, Dellon AL. Anatomic variability of the ilioinguinal and genitofemoral nerve: implications for the treatment of groin pain. Plast Reconstr Surg 2001; 108: 1618-23

2 Fuchs EF. Cord block anesthesia for scrotal surgery. J Urol 1982; 128: $718-9$

3 Issa MM, Hsiao K, Bassel YS, Bouet R, Young MR, Petros JA. Spermatic cord anesthesia block for scrotal procedures in outpatient clinic setting. J Urol 2004; 172: 2358-61

4 Kaye KW, Lange PH, Fraley EE. Spermatic cord block in urologic surgery. J Urol 1982; 128: 720-1

5 Cassady JF Jr. Regional anesthesia for urologic procedures. Urol Clin North Am 1987; 14: 43-50

6 Ezeh UI, Shepherd S, Moore HD, Cooke ID. Morbidity and costeffectiveness analysis of outpatient analgesia versus general anaesthesia for testicular sperm extraction in men with azoospermia due to defects in spermatogenesis. Hum Reprod 1999; 14: 321-8

7 Nordin P, Zetterstrom H, Gunnarsson U, Nilsson E. Local, regional, or general anaesthesia in groin hernia repair: multicentre randomised trial. Lancet 2003; 362: 853-8

8 Kaye KW, Gonzalez R, Fraley EE. Microsurgical vasovasostomy: an outpatient procedure under local anesthesia. J Urol 1983; 129: 992-4

9 Lorenz ST, Renkawitz K. Spermatic cord block and periscrotal block according to Reclus. Alternative procedure for critically ill patients. Anaesthesist 2008; 57: 893-7

10 Wakefield SE, Elewa AA. Spermatic cord block: a safe technique for intrascrotal surgery. Ann R Coll Surg Engl 1994; 76: 401-2

11 Goldstein M, Young GPH, Einer-Jensen N. Testicular artery damage due to infiltration with a fine-gauge needle: experimental evidence suggesting that blind spermatic cord blockade should be abandoned. Surg Forum 1983; 24: 653-6

12 Abrahams MS, Aziz MF, Fu RF, Horn JL. Ultrasound guidance compared with electrical neurostimulation for peripheral nerve block: a systematic review and meta-analysis of randomized controlled trials. Br J Anaesth 2009; 102: 408-17

13 Marhofer P, Harrop-Griffiths W, Kettner SC, Kirchmair L. Fifteen years of ultrasound guidance in regional anaesthesia: part $1 . \mathrm{Br}$ J Anaesth 2010; 104: 538-46

14 Casati A, Danelli G, Baciarello $M$, et al. A prospective, randomized comparison between ultrasound and nerve stimulation guidance for multiple injection axillary brachial plexus block. Anesthesiology 2007; 106: 992-6 